\title{
Posttraumatic Stress Disorder Checklist for DSM-5 (PCL-5): transcultural adaptation of the Brazilian version
}

\author{
Flávia L. Osório1,2, Thiago Dornela Apolinario da Silva1, Rafael Guimarães dos Santosi1,2, Marcos Hortes N. Chagas², \\ Natalia Mota S. Chagas ${ }^{1}$, Rafael Faria Sanches ${ }^{1}$, José Alexandre de Souza Crippa ${ }^{1,2}$
}

1 Department of Neurosciences and Behavior, Ribeirão Preto Medical School, University of São Paulo (FMRP-USP), Ribeirão Preto, SP, Brazil.
${ }^{2}$ National Institute of Science and Technology - Translational Medicine, Ribeirão Preto, SP, Brazil.
${ }^{3}$ Center of Biological and Health Sciences, Federal University of São Carlos (UFSCar), São CarloS, SP, Brazil.

Institution where the study was conducted: Department of Neurosciences and Behavior, Ribeirão Preto Medical School, University of São Paulo, Ribeirão Preto.

Received: 8/16/2016 - Accepted: 12/8/2016

DOI: 10.1590/0101-60830000000107

\begin{abstract}
Background: DSM-5 introduced some modifications on Posttraumatic Stress Disorder (PTSD) criteria. The instruments developed for the assessment of aspects related to PTSD needed a reformulation, as was the case of the Posttraumatic Stress Disorder Checklist (PCL). Objectives: To present the process of transcultural adaptation of the three forms of the PCL-5 to Brazilian Portuguese, as well as its face validity. Methods: The procedure involved independent translations, a synthesis version, back translation by an independent translator, evaluation by the original author, analysis by an expert committee, and a pretesting study (10 subjects with/without experience of a traumatic situation). The last two steps formed the face validity procedure. Results: The synthesis version was approved by the original author and the agreement percentage by the expert committee was excellent, with only two items showing $<90 \%$. The pretesting study showed that the Brazilian version was well understood and linguistically and culturally accepted by the participants, which did not make significant suggestions for changes. Discussion: Transcultural adaptation of the PCL-5 for Brazilian Portuguese followed a rigid and standardized procedure. Therefore, after having its face validity assessed by an expert committee and by the target population, it is apt to be used.
\end{abstract}

Osório FL et al. / Arch Clin Psychiatry. 2017;44(1):10-9

Keywords: PCL-5, transcultural adaptation, trauma, stress, scale.

\section{Introduction}

The $5^{\text {th }}$ edition of the Diagnostic and Statistical Manual of Mental Disorders (DSM-5), released on May 2013, introduced modifications in the different diagnostic categories, involving inclusion, reformulation, and exclusion of diagnosis. Regarding especially Posttraumatic Stress Disorder (PTSD), this disorder no longer belongs to the group of anxiety disorders and was included in a new chapter with five different disorders in which their origin can be specifically attributed to stressful and traumatic situations (Trauma- and Stress-Related Disorders) ${ }^{1}$.

Moreover, some modifications were made on PTSD criteria ${ }^{1}$. Considering these modifications, the instruments developed so far for the assessment of aspects related to PTSD needed a reformulation, as was the case of the Posttraumatic Stress Disorder Checklist (PCL), one of the most used instruments in the area $^{2}$ that aims to screen subjects with PTSD, make a provisional PTSD diagnosis, and to monitor symptomatic changes during/after treatment.

The PCL was initially proposed by Weathers ${ }^{3}$ and Weathers et al. ${ }^{4}$ in three versions (civilian, military and specific) composed by 17 items each, related to the diagnostic criteria of the DSM-III/ DSM-IV and scored in a Likert scale from 1 (not at all) to 5 (extremely).

The current version ${ }^{2}$, reformulated in several aspects, was denominated PCL-5 and is composed of 20 items. The main modifications of this new version include: a) availability of a single version in three different forms (without Criterion A, plus Criterion A, plus Life Events Checklist for DSM-5 (LEC-5) 5 and Criterion A); b) creation and adjustment of the items to current symptoms and diagnostic criteria (only nine items remained unchanged and three new items were included); c) new rating scale (0: not at all -4 : extremely).
The PCL-5 can be interpreted in several distinct ways: a) total severity score; b) cluster severity scores; c) provisional PTSD diagnosis; d) cut-point suggestions (obtained through specific studies of psychometric validation already established in different contexts of the North American (military service members ${ }^{6}$, veterans ${ }^{7,8}$, trauma exposed college students ${ }^{2}$, community sample of adults ${ }^{8}$ ) and Swedish (parents of children with burns ${ }^{9}$ ) realities.

Thus, considering the importance of this instrument for PTSDrelated studies worldwide, it is essential that it should be translated, tested, and made available for use in the Brazilian context, as was the case of the PCL- $4^{10}$.

The objective of the present study is to report the process of transcultural adaptation of the PCL-5 for the Brazilian Portuguese in its three forms and of its face validity.

\section{Methods}

Initially, authorization to perform the study was obtained after establishing contact by electronic mail with the group that holds the instrument's copyright (National Center for PTSD).

The procedure used to perform the transcultural adaptation of the PCL- 5 was based in the stages proposed by Beaton et al. ${ }^{11}$, which involve translations by specialized and non-specialized bilingual translators, a synthesis version, back translation by an independent translator, evaluation by the original author, analysis by an expert committee (consensus version), and pretesting study. These last stages constitute the face validity procedure.

The expert committee had the role of judging, individually, the synthesis version previously approved by the original author according to different types of equivalence in relation to the original version. For conceptual equivalence, the terms used along the process 
of adaptation were analyzed for correspondence with the concepts of the original version. For semantic equivalence, the meaning (grammatical and vocabulary) of the terms of the two version was compared. For idiomatic equivalence, the correspondence between the referential (denotative) and literal meaning of the terms/expressions of both versions was analyzed. Finally, for the experimental or cultural equivalence the coherence and compatibility of the terms with the Brazilian context were analyzed ${ }^{12}$.

After this stage, the researcher responsible for the development of the synthesis version performed a concordance analysis of the evaluation done by the experts by calculating the agreement percentage (ratio between the number of evaluators that agreed with the equivalences and the total number of evaluators, multiplied by 100). After this analysis, the final version was developed including the new alterations.

To conclude the process of transcultural adaption a pretesting study was performed by applying the final consensus version to 10 subjects with and without experience with a traumatic situation. Individual interviews were performed to assess scale comprehension, and the subjects were asked to readand later to paraphrase the scale's instructions, items, and answer options.

\section{Results and discussion}

The independent translations from English to Portuguese were performed by an English teacher and two psychiatrists with different lines of work/investigation. The synthesis version was prepared by the first author (FLO), who has a long experience in the validation of this kind of instruments, and the back translation was conducted by a bilingual neuroscientist (RGS) and then was evaluated by one of members of National Center for PTSD (Matthew Yoder), being considered adequate.

The expert committee was composed by three psychiatrists with long clinical experience in anxiety (JASC/RFS) and trauma (TA) related disorders.

Table 1 shows the distribution of the agreement percentage from the expert committee regarding the synthesis version, for the different aspect of the validated instrument.

As can be observed in Table 1, the agreement percentage was excellent, with only two items with the percentage inferior to $90 \%$. Despite this, since the expert suggestions were highly appropriate and according to the denominations used in the Brazilian version of the DSM-5, all of them were accepted and are described below.

For the LEC-5 instructions there were two disagreements. First, the expression "learned about it" was initially translated to "ouviu sobre", but for idiomatic reasons the term suggested was "ficou sabendo". The same suggestion was made and included for the answer to question 3. Second, the words "police" and "military" were initially translated to "polícia" and "militar", but for conceptual reasons they were changed to "polícia civil" and "polícia militar".

Still regarding the LEC-5, the item 1 showed a disagreement regarding cultural equivalence. According to the expert opinion, the example "deslizamento de terra" was included, since this kind of natural disaster is very frequent in our context. In the item 4, the expression "recreational activity" was adjusted from "atividade recreativa" to "atividade de lazer", and in the item 9 the words "uncomfortable/unwanted" were initially translated to "desconfortável/indesejada" but for conceptual reasons were changed to "constrangedora/não consentida".

Criterion A was evaluated in its totality, and three suggestions were made. Two of them were previously described (regarding the expression "learned about it" and the words "police" and "military") and were also incorporated. The third disagreement referred to the term "checked", which was denominated in the synthesis version as "marcou". It was suggested to change the term for the word "assinalou", which is more adequate to our context.
About the instrument itself, in the items 1 and 6 the experts suggested to translate the word "memories" as "lembranças" and not "memórias", and in the item 7 the word "relacionadas" was included in the sentence "Evitar lembranças externas relacionadas à situação estressante" for semantic reasons.

In the items 8 and 10 there were two disagreements. First, the translation of the expression "trouble remembering" as "dificuldade para lembrar" was modified to "não conseguir lembrar". Second, in the item 10, the translation of the expression "someone else" was changed from "alguém" to "aos outros". Finally, in the item 13, the translation of the expression "cut off" was changed from "afastado" to "isolado" for idiomatic reasons.

After the conclusion of the consensus version and the introduction of the above mentioned suggestions, a pretesting study was performed with 10 subjects: four ambulatory patients from a psychiatric unit with PTSD diagnosis, and six subjects from the general population. Subjects ( 5 men $/ 5$ women) had a mean age of 41.5 years $(\mathrm{dp}=15.07)$ and different educational backgrounds $(20 \%$ elementary school, $40 \%$ high school, and $40 \%$ higher education).

Subjects did not show signs of discomfort and/or lack of motivation during the application of the scale.

The Brazilian version of the PCL-5 was well understood and accepted both linguistically and culturally by the sample, which did not make any suggestions for significant changes. Minor adjustments were made only in the item B of part 2 from Criterion A, which included adding the information "(item 1 a 17$)$ " in a parenthesis after the affirmative "Se você vivenciou mais de um dos eventos da Parte 1", and underlying the information "mais incomoda" to facilitate both comprehension and evaluation by the subject.

The layout of the final version was maintained, and the transcultural adaptation of the PCL-5 for the Brazilian context was concluded. The instrument was denominated "Lista de Verificação do TEPT para o DSM-5 (PCL-5)" and can be found in the Annex in its three forms.

It should be noted that the form "Sem Critério A" (Annex 1) contains only a brief instruction and the 20 items that compose the instrument, and that it is appropriate to use when the experienced trauma has been evaluated by others means. The form "Com Critério A" (Annex 2) contains the definition of criterion A and examples of events that the subject could have experienced. The subject is asked to identify his/her worse fear and to evaluate if it fulfills criterion A. The form "Com lista de eventos de vida para o DSM-5 e Critério A" (Annex 3) contains the LEC-5, as well as the most detailed evaluation of criterion A. The last two forms should be used when there is a necessity to evaluate the Criterion A.

\section{Conclusion}

The transcultural adaptation of the PCL-5 for the Brazilian Portuguese followed a rigid and standardized protocol, having its face validity assessed by an expert group and by the target population.

The availability of this new version of the instrument for the Brazilian clinical and research context is highly important, since it is one of the most used instruments worldwide to assess PTSD, being adapted to different languages and cultures.

The PCL-5, in its three forms, can be freely used in the target population. Currently it is being psychometrically analyzed by our group, in clinical and non-clinical populations.

\section{Conflicts of interests}

There is no conflict of interest.

\section{Research support}

There is no research support. 
Table 1. Agreement percentage from the expert committee regarding the brief version $(N=3)$

\begin{tabular}{|c|c|c|c|c|c|c|c|c|c|c|}
\hline \multirow{3}{*}{ Assessed structure } & \multicolumn{10}{|c|}{ Agreement Percentage/Equivalence } \\
\hline & \multicolumn{2}{|c|}{ Semantic } & \multicolumn{2}{|c|}{ Idiomatic } & \multicolumn{2}{|c|}{ Cultural } & \multicolumn{2}{|c|}{ Conceptual } & \multicolumn{2}{|c|}{ Mean by item } \\
\hline & $\mathrm{N}$ & $\%$ & $\mathrm{~N}$ & $\%$ & $\mathrm{~N}$ & $\%$ & $\mathrm{~N}$ & $\%$ & $\mathrm{~N}$ & $\%$ \\
\hline Title & 3 & 100 & 3 & 100 & 3 & 100 & 3 & 100 & 3 & 100 \\
\hline Instruction-LEC5 - Part 1 & 3 & 100 & 1 & 33 & 3 & 100 & 2 & 67 & 2.3 & 75 \\
\hline Item 1- LEC5 & 3 & 100 & 3 & 100 & 2 & 67 & 3 & 100 & 2.8 & 91.8 \\
\hline Item 2- LEC5 & 3 & 100 & 3 & 100 & 3 & 100 & 3 & 100 & 3 & 100 \\
\hline Item 3- LEC5 & 3 & 100 & 3 & 100 & 3 & 100 & 3 & 100 & 3 & 100 \\
\hline Item 4- LEC5 & 3 & 100 & 2 & 67 & 3 & 100 & 3 & 100 & 2.8 & 91.8 \\
\hline Item 5- LEC5 & 3 & 100 & 3 & 100 & 3 & 100 & 3 & 100 & 3 & 100 \\
\hline Item 6- LEC5 & 3 & 100 & 3 & 100 & 3 & 100 & 3 & 100 & 3 & 100 \\
\hline Item 7- LEC5 & 3 & 100 & 3 & 100 & 3 & 100 & 3 & 100 & 3 & 100 \\
\hline Item 8- LEC5 & 3 & 100 & 3 & 100 & 3 & 100 & 3 & 100 & 3 & 100 \\
\hline Item 9- LEC5 & 3 & 100 & 3 & 100 & 3 & 100 & 2 & 67 & 2.8 & 91.8 \\
\hline Item 10- LEC5 & 3 & 100 & 3 & 100 & 3 & 100 & 3 & 100 & 3 & 100 \\
\hline Item 11- LEC5 & 3 & 100 & 3 & 100 & 3 & 100 & 3 & 100 & 3 & 100 \\
\hline Item 12- LEC5 & 3 & 100 & 3 & 100 & 3 & 100 & 3 & 100 & 3 & 100 \\
\hline Item 13- LEC5 & 3 & 100 & 3 & 100 & 3 & 100 & 3 & 100 & 3 & 100 \\
\hline Item 14- LEC5 & 3 & 100 & 3 & 100 & 3 & 100 & 3 & 100 & 3 & 100 \\
\hline Item 15- LEC5 & 3 & 100 & 3 & 100 & 3 & 100 & 3 & 100 & 3 & 100 \\
\hline Item 16- LEC5 & 3 & 100 & 3 & 100 & 3 & 100 & 3 & 100 & 3 & 100 \\
\hline Item 17- LEC5 & 3 & 100 & 3 & 100 & 3 & 100 & 3 & 100 & 3 & 100 \\
\hline Option answer 1- LEC5 & 3 & 100 & 3 & 100 & 3 & 100 & 3 & 100 & 3 & 100 \\
\hline Option answer 2- LEC5 & 3 & 100 & 3 & 100 & 3 & 100 & 3 & 100 & 3 & 100 \\
\hline Option answer 3- LEC5 & 3 & 100 & 1 & 33 & 3 & 100 & 3 & 100 & 2.5 & 83.5 \\
\hline Option answer 4- LEC5 & 3 & 100 & 3 & 100 & 3 & 100 & 3 & 100 & 3 & 100 \\
\hline Option answer 5- LEC5 & 3 & 100 & 3 & 100 & 3 & 100 & 3 & 100 & 3 & 100 \\
\hline Option answer 6- LEC5 & 3 & 100 & 3 & 100 & 3 & 100 & 3 & 100 & 3 & 100 \\
\hline Mean/equivalence - LEC5 & 3 & 100 & 2.8 & 93 & 2.95 & 98.3 & 2.91 & 97 & - & - \\
\hline Mean/total scale - LEC5 & - & - & - & - & - & - & - & - & 2.92 & 97.3 \\
\hline Criterion A - Part 2 & 3 & 100 & 1 & 33 & 3 & 100 & 2 & 67 & 2.3 & 75 \\
\hline Instruções-PCL-5 - Part 3 & 3 & 100 & 3 & 100 & 3 & 100 & 3 & 100 & 3 & 100 \\
\hline Item 1- PCL5 & 2 & 67 & 3 & 100 & 3 & 100 & 3 & 100 & 2.8 & 91.8 \\
\hline Item 2- PCL5 & 3 & 100 & 3 & 100 & 3 & 100 & 3 & 100 & 3 & 100 \\
\hline Item 3- PCL5 & 3 & 100 & 3 & 100 & 3 & 100 & 3 & 100 & 3 & 100 \\
\hline Item 4- PCL5 & 3 & 100 & 3 & 100 & 3 & 100 & 3 & 100 & 3 & 100 \\
\hline Item 5- PCL5 & 3 & 100 & 3 & 100 & 3 & 100 & 3 & 100 & 3 & 100 \\
\hline Item 6- PCL5 & 2 & 67 & 3 & 100 & 3 & 100 & 3 & 100 & 2.8 & 91.8 \\
\hline Item 7- PCL5 & 2 & 67 & 3 & 100 & 3 & 100 & 3 & 100 & 2.8 & 91.8 \\
\hline Item 8- PCL5 & 2 & 67 & 3 & 100 & 3 & 100 & 3 & 100 & 2.8 & 91.8 \\
\hline Item 9- PCL5 & 3 & 100 & 3 & 100 & 3 & 100 & 3 & 100 & 3 & 100 \\
\hline Item 10- PCL5 & 2 & 67 & 3 & 100 & 3 & 100 & 3 & 100 & 2.8 & 91.8 \\
\hline Item 11- PCL5 & 3 & 100 & 3 & 100 & 3 & 100 & 3 & 100 & 3 & 100 \\
\hline Item 12- PCL5 & 3 & 100 & 3 & 100 & 3 & 100 & 3 & 100 & 3 & 100 \\
\hline Item 13- PCL5 & 3 & 100 & 2 & 67 & 3 & 100 & 3 & 100 & 2.8 & 91.8 \\
\hline Item 14- PCL5 & 3 & 100 & 3 & 100 & 3 & 100 & 3 & 100 & 3 & 100 \\
\hline Item 15- PCL5 & 3 & 100 & 3 & 100 & 3 & 100 & 3 & 100 & 3 & 100 \\
\hline Item 16- PCL5 & 3 & 100 & 3 & 100 & 3 & 100 & 3 & 100 & 3 & 100 \\
\hline Item 17- PCL5 & 3 & 100 & 3 & 100 & 3 & 100 & 3 & 100 & 3 & 100 \\
\hline Item 18- PCL5 & 3 & 100 & 3 & 100 & 3 & 100 & 3 & 100 & 3 & 100 \\
\hline Item 19- PCL5 & 3 & 100 & 3 & 100 & 3 & 100 & 3 & 100 & 3 & 100 \\
\hline Item 20- PCL5 & 3 & 100 & 3 & 100 & 3 & 100 & 3 & 100 & 3 & 100 \\
\hline Option answer 1- PCL5 & 3 & 100 & 3 & 100 & 3 & 100 & 3 & 100 & 3 & 100 \\
\hline Option answer 2- PCL5 & 3 & 100 & 3 & 100 & 3 & 100 & 3 & 100 & 3 & 100 \\
\hline Option answer 3- PCL5 & 3 & 100 & 3 & 100 & 3 & 100 & 3 & 100 & 3 & 100 \\
\hline Option answer 4- PCL5 & 3 & 100 & 3 & 100 & 3 & 100 & 3 & 100 & 3 & 100 \\
\hline Option answer 5- PCL5 & 3 & 100 & 3 & 100 & 3 & 100 & 3 & 100 & 3 & 100 \\
\hline \begin{tabular}{|l|} 
Mean/equivalence PCL5 \\
\end{tabular} & 2.8 & 93.3 & 2.96 & 98.7 & 3 & 100 & 3 & 100 & - & - \\
\hline Mean/total scale PCL5 & - & - & - & - & - & - & - & - & 2.95 & 98.3 \\
\hline
\end{tabular}




\section{References}

1. Araújo AC, Lotufo Neto F. The new North American classification of Mental Disorders - DSM-5. Rev Bras Ter Comport Cogn. 2014;16(1):67-82.

2. Blevins CA, Weathers FW, Davis MT, Witte TK, Domino JL. The Posttraumatic Stress Disorder Checklist for DSM-5 (PCL-5): Development and initial psychometric evaluation. J Trauma Stress. 2015;28:489-98.

3. Weathers FW. Posttraumatic Stress Disorder Cheklist. In: Reyes G, Elhai JD, Ford JD, editors. Encyclopedia of psychological trauma. Hoboken, NJ: Wiley; 2008. p. 491-4.

4. Weathers FW, Litz BT, Herman DS, Huska JA, Keane TM. The PTSD Checklist: reliability, validity, and diagnostic utility. Paper presented at the annual meeting of the International Society for Traumatic Stress Studies, San Antonio, TX. 1993.

5. Weathers FW, Blake DD, Schnurr PP, Kaloupek DG, Marx BP, Keane TM. The Life Events Checklist for DSM-5 (LEC-5). Instrument available from the National Center for PTSD. Available at: <www.ptsd.va.gov>.

6. Wortmann JH, Jordan AH, Weathers FW, Resick PA, Dondanville KA, Hall-Clark B, et al. Psychometric analysis of the PTSD Checklist-5 (PCL-5) among treatment-seeking military service members. Psychol Assess. 2016;28(11):1392-403.
7. Bovin MJ, Marx BP, Weathers FW, Gallagher MW, Rodriguez P, Schnurr PP, et al. Psychometric properties of the PTSD Checklist for Diagnostic and Statistical Manual of Mental Disorders-Fifth Edition (PCL-5) in veterans. Psychol Assess. 2016;28(11):1379-91.

8. Price M, Szafranski DD, van Stolk-Cooke K, Gros DF. Investigation of abbreviated 4 and 8 item versions of the PTSD Checklist 5. Psychiatry Res. 2016;30;239:124-30.

9. Sveen J, Bondjers K, Willebrand M. Psychometric properties of the PTSD Checklist for DSM-5: a pilot study. Eur J Psychotraumatol. 2016;7: 30165.

10. Berger W, Mendlowicz MV, Souza WF, Figueira I. Semantic equivalence of the Portuguese version of the Post-Traumatic Stress Disorder Checklist - Civilian Version (PCL-C) for the screening of post-traumatic stress disorder. Rev Psiquiatr Rio Gd Sul. 2004;26(2):167-75.

11. Beaton DE, Bombardier C, Guillemin F, Ferraz MB. Guidelines for the Process of Cross-Cultural Adaptation of Self-Report Measures. Spine. 2000;25(24):3186-91.

12. Ciconelli RM, Ferraz MB, Santos W, Meinão I, Quaresma MR. BrazilianPortuguese version of the SF-36. A reliable and valid quality of life outcome measure. Rev Bras Reumatol. 1999;39(3):143-50. 
Annex 1

\section{Lista de verificação do TEPT para o DSM-5}

\section{Versão autorizada, traduzida e adaptada para o português do Brasil em junho 2016}

Autores: Flávia de Lima Osório, Marcos N. Hortes Chagas, Natalia M. Souza, Rafael Guimarães dos Santos, Thiago Dornela Apolinario da Silva, Rafael Faria Sanches, José Alexandre de Souza Crippa

Versão Original: The PTSD Checklist for DSM-5 with Life Events Checklist for DSM-5 and Criterion A. Reference: Weathers FW, Litz BT, Keane TM, Palmieri PA, Marx BP, Schnurr PP. (2013). The PTSD Checklist for DSM-5 (PCL-5)- Extended Criterion A (Mesasurement instrument)

\section{PCL-5}

Abaixo há uma lista de problemas que as pessoas às vezes apresentam em resposta a uma experiência muito estressante. Por favor, leia cuidadosamente cada problema e então circule um dos números à direita para indicar o quanto você tem sido incomodado por este problema no último mês.

\begin{tabular}{|c|c|c|c|c|c|}
\hline No último mês, quanto você foi incomodado por: & $\begin{array}{l}\text { De modo } \\
\text { nenhum }\end{array}$ & Um pouco & $\begin{array}{l}\text { Modera- } \\
\text { damente }\end{array}$ & Muito & Extremamente \\
\hline 1. Lembranças indesejáveis, perturbadoras e repetitivas da experiência estressante? & 0 & 1 & 2 & 3 & 4 \\
\hline 2. Sonhos perturbadores e repetitivos com a experiência estressante? & 0 & 1 & 2 & 3 & 4 \\
\hline $\begin{array}{l}\text { 3. De repente, sentindo ou agindo como se a experiência estressante estivesse, de } \\
\text { fato, acontecendo de novo (como se você estivesse revivendo-a, de verdade, lá no } \\
\text { passado)? }\end{array}$ & 0 & 1 & 2 & 3 & 4 \\
\hline 4. Sentir-se muito chateado quando algo lembra você da experiência estressante? & 0 & 1 & 2 & 3 & 4 \\
\hline $\begin{array}{l}\text { 5. Ter reações físicas intensas quando algo lembra você da experiência estressante } \\
\text { (por exemplo, coração apertado, dificuldades para respirar, suor excessivo)? }\end{array}$ & 0 & 1 & 2 & 3 & 4 \\
\hline $\begin{array}{l}\text { 6. Evitar lembranças, pensamentos, ou sentimentos relacionados à experiência } \\
\text { estressante? }\end{array}$ & 0 & 1 & 2 & 3 & 4 \\
\hline $\begin{array}{l}\text { 7. Evitar lembranças externas da experiência estressante (por exemplo, pessoas, } \\
\text { lugares, conversas, atividades, objetos ou situações)? }\end{array}$ & 0 & 1 & 2 & 3 & 4 \\
\hline 8. Não conseguir se lembrar de partes importantes da experiência estressante? & 0 & 1 & 2 & 3 & 4 \\
\hline $\begin{array}{l}\text { 9. Ter crenças negativas intensas sobre você, outras pessoas ou o mundo (por } \\
\text { exemplo, ter pensamentos tais como: "Eu sou ruim", "existe algo seriamente errado } \\
\text { comigo", "ninguém é confiável", "o mundo todo é perigoso")? }\end{array}$ & 0 & 1 & 2 & 3 & 4 \\
\hline $\begin{array}{l}\text { 10. Culpar a si mesmo ou aos outros pela experiência estressante ou pelo que } \\
\text { aconteceu depois dela? }\end{array}$ & 0 & 1 & 2 & 3 & 4 \\
\hline 11. Ter sentimentos negativos intensos como medo, pavor, raiva, culpa ou vergonha? & 0 & 1 & 2 & 3 & 4 \\
\hline 12. Perder 0 interesse em atividades que você costumava apreciar? & 0 & 1 & 2 & 3 & 4 \\
\hline 13. Sentir-se distante ou isolado das outras pessoas? & 0 & 1 & 2 & 3 & 4 \\
\hline $\begin{array}{l}\text { 14. Dificuldades para vivenciar sentimentos positivos (por exemplo, ser incapaz de } \\
\text { sentir felicidade ou sentimentos amorosos por pessoas próximas a você)? }\end{array}$ & 0 & 1 & 2 & 3 & 4 \\
\hline 15. Comportamento irritado, explosões de raiva ou agir agressivamente? & 0 & 1 & 2 & 3 & 4 \\
\hline 16. Correr muitos riscos ou fazer coisas que podem Ihe causar algum mal? & 0 & 1 & 2 & 3 & 4 \\
\hline 17. Ficar "super" alerta, vigilante ou de sobreaviso? & 0 & 1 & 2 & 3 & 4 \\
\hline 18. Sentir-se apreensivo ou assustado facilmente? & 0 & 1 & 2 & 3 & 4 \\
\hline 19. Ter dificuldades para se concentrar? & 0 & 1 & 2 & 3 & 4 \\
\hline 20. Problemas para adormecer ou continuar dormindo? & 0 & 1 & 2 & 3 & 4 \\
\hline
\end{tabular}




\section{Annex 2}

\section{Lista de verificação do TEPT para o DSM-5 com Critério A}

Versão autorizada, traduzida e adaptada para o português do Brasil em junho 2016

Autores: Flávia de Lima Osório, Marcos N. Hortes Chagas, Natalia M. Souza, Rafael Guimarães dos Santos, Thiago Dornela Apolinario da Silva, Rafael Faria Sanches, José Alexandre de Souza Crippa

Versão Original: The PTSD Checklist for DSM-5 with Life Events Checklist for DSM-5 and Criterion A. Reference: Weathers FW, Litz BT, Keane TM, Palmieri PA, Marx BP, Schnurr PP. (2013). The PTSD Checklist for DSM-5 (PCL-5)- Extended Criterion A (Mesasurement instrument)

\section{PCL-5 com Critério A}

Instruções: Este questionário pergunta sobre problemas que você possa ter tido após uma experiência muito estressante envolvendo morte real ou ameaça de morte, ferimentos graves ou violência sexual. Estas experiências podem ser algo que aconteceu diretamente com você, algo que você testemunhou, ou algo que você ficou sabendo ter acontecido com um familiar próximo ou amigo próximo. Alguns exemplos são um grave acidente, incêndio, catástrofes como um furacão, tornado ou tremor/ deslizamento de terra; agressão ou abuso físico ou sexual; guerra; homicídio; ou suicídio.

Em primeiro lugar, por favor, responda a algumas perguntas sobre o seu pior evento, o qual, para este questionário, significa o evento que mais incomoda você neste momento. Este evento pode ser um dos exemplos acima ou alguma outra experiência muito estressante. Também pode ser um evento único (por exemplo, um acidente de carro) ou vários eventos semelhantes (por exemplo, vários eventos estressantes em uma zona de guerra ou abuso sexual repetido).

Resumidamente identifique o pior evento (se você se sentir confortável para fazer isto):

Há quanto tempo isso aconteceu? (por favor, faça uma estimativa se você não tem certeza)

Envolveu morte real ou ameaça de morte, ferimentos graves ou violência sexual?

Sim

- Não

Como você vivenciou este evento?

Aconteceu comigo diretamente

__ Eu testemunhei este evento

Eu fiquei sabendo que o evento aconteceu com um membro próximo da família ou amigo próximo

__ Eu fui exposto repetidamente a detalhes deste evento como parte do meu trabalho (por exemplo, paramédico, policial civil, militar ou outro socorrista)

_ Outros, por favor descreva:

Se o evento envolveu a morte de um membro próximo da família ou amigo próximo, foi devido a algum tipo de acidente ou violência, ou foi devido a causas naturais?

__ Acidente ou violência

Causas naturais

_ Não se aplica (0 evento não envolveu a morte de um membro próximo da família ou amigo próximo) 
Em segundo lugar, veja abaixo uma lista de problemas que as pessoas às vezes apresentam em resposta a uma experiência muito estressante. Pensando em seu pior evento, por favor, leia cuidadosamente cada problema e então circule um dos números à direita para indicar o quanto você tem sido incomodado por este problema no último mês.

\begin{tabular}{|c|c|c|c|c|c|}
\hline No último mês, quanto você foi incomodado por: & De modo nenhum & Um pouco & Moderadamente & Muito & Extremamente \\
\hline $\begin{array}{l}\text { 1. Lembranças indesejáveis, perturbadoras e repetitivas da experiência } \\
\text { estressante? }\end{array}$ & 0 & 1 & 2 & 3 & 4 \\
\hline 2. Sonhos perturbadores e repetitivos com a experiência estressante? & 0 & 1 & 2 & 3 & 4 \\
\hline $\begin{array}{l}\text { 3. De repente, sentindo ou agindo como se a experiência estressante estivesse, } \\
\text { de fato, acontecendo de novo (como se você estivesse revivendo-a, de verdade, } \\
\text { lá no passado)? }\end{array}$ & 0 & 1 & 2 & 3 & 4 \\
\hline $\begin{array}{l}\text { 4. Sentir-se muito chateado quando algo lembra você da experiência } \\
\text { estressante? }\end{array}$ & 0 & 1 & 2 & 3 & 4 \\
\hline $\begin{array}{l}\text { 5. Ter reações físicas intensas quando algo lembra você da experiência } \\
\text { estressante (por exemplo, coração apertado, dificuldades para respirar, suor } \\
\text { excessivo)? }\end{array}$ & 0 & 1 & 2 & 3 & 4 \\
\hline $\begin{array}{l}\text { 6. Evitar lembranças, pensamentos, ou sentimentos relacionados à experiência } \\
\text { estressante? }\end{array}$ & 0 & 1 & 2 & 3 & 4 \\
\hline $\begin{array}{l}\text { 7. Evitar lembranças externas da experiência estressante (por exemplo, pessoas, } \\
\text { lugares, conversas, atividades, objetos ou situações)? }\end{array}$ & 0 & 1 & 2 & 3 & 4 \\
\hline 8. Não conseguir se lembrar de partes importantes da experiência estressante? & 0 & 1 & 2 & 3 & 4 \\
\hline $\begin{array}{l}\text { 9. Ter crenças negativas intensas sobre você, outras pessoas ou o mundo (por } \\
\text { exemplo, ter pensamentos tais como: "Eu sou ruim", "existe algo seriamente } \\
\text { errado comigo", "ninguém é confiável", "o mundo todo é perigoso")? }\end{array}$ & 0 & 1 & 2 & 3 & 4 \\
\hline $\begin{array}{l}\text { 10. Culpar a si mesmo ou aos outros pela experiência estressante ou pelo que } \\
\text { aconteceu depois dela? }\end{array}$ & 0 & 1 & 2 & 3 & 4 \\
\hline $\begin{array}{l}\text { 11. Ter sentimentos negativos intensos como medo, pavor, raiva, culpa ou } \\
\text { vergonha? }\end{array}$ & 0 & 1 & 2 & 3 & 4 \\
\hline 12. Perder 0 interesse em atividades que você costumava apreciar? & 0 & 1 & 2 & 3 & 4 \\
\hline 13. Sentir-se distante ou isolado das outras pessoas? & 0 & 1 & 2 & 3 & 4 \\
\hline $\begin{array}{l}\text { 14. Dificuldades para vivenciar sentimentos positivos (por exemplo, ser incapaz } \\
\text { de sentir felicidade ou sentimentos amorosos por pessoas próximas a você)? }\end{array}$ & 0 & 1 & 2 & 3 & 4 \\
\hline 15. Comportamento irritado, explosões de raiva ou agir agressivamente? & 0 & 1 & 2 & 3 & 4 \\
\hline 16. Correr muitos riscos ou fazer coisas que podem Ihe causar algum mal? & 0 & 1 & 2 & 3 & 4 \\
\hline 17. Ficar "super" alerta, vigilante ou de sobreaviso? & 0 & 1 & 2 & 3 & 4 \\
\hline 18. Sentir-se apreensivo ou assustado facilmente? & 0 & 1 & 2 & 3 & 4 \\
\hline 19. Ter dificuldades para se concentrar? & 0 & 1 & 2 & 3 & 4 \\
\hline 20. Problemas para adormecer ou continuar dormindo? & 0 & 1 & 2 & 3 & 4 \\
\hline
\end{tabular}


Annex 3

\section{Lista de verificação do TEPT para o DSM-5 com Lista de eventos de vida para o DSM-5 e Critério A}

\section{Versão autorizada, traduzida e adaptada para o português do Brasil em junho 2016}

Autores: Flávia de Lima Osório, Marcos N. Hortes Chagas, Natalia M. Souza, Rafael Guimarães dos Santos, Thiago Dornela Apolinario da Silva, Rafael Faria Sanches, José Alexandre de Souza Crippa

Versão Original: The PTSD Checklist for DSM-5 with Life Events Checklist for DSM-5 and Criterion A. Reference: Weathers FW, Litz BT, Keane TM, Palmieri PA, Marx BP, Schnurr PP. (2013). The PTSD Checklist for DSM-5 (PCL-5)- Extended Criterion A (Mesasurement instrument)

\section{PCL-5 com LEV-5 e Critério A}

\section{Parte 1}

Instruções: Abaixo estão listadas um número de situações estressantes ou difíceis que algumas vezes acontecem com as pessoas. Para cada evento, marque um ou mais itens à direita para indicar que: (a) aconteceu com você pessoalmente; (b) você testemunhou o evento, que aconteceu com outra pessoa; (c) você ficou sabendo a respeito do evento, o qual aconteceu com um familiar ou amigo próximo; (d) você foi exposto ao evento como parte de seu trabalho (por exemplo, paramédico, polícia civil, militar ou outro socorrista); (e) você não tem certeza se o evento se enquadra; ou (f) não se aplica a você.

Certifique-se de considerar toda sua vida (desde a infância até a vida adulta) quando você examinar a lista de eventos.

\begin{tabular}{|c|c|c|c|c|c|c|}
\hline Evento & $\begin{array}{l}\text { Aconteceu } \\
\text { comigo }\end{array}$ & Testemunhei & $\begin{array}{l}\text { Fiquei } \\
\text { sabendo }\end{array}$ & $\begin{array}{l}\text { Parte do meu } \\
\text { trabalho }\end{array}$ & $\begin{array}{l}\text { Não tenho } \\
\text { certeza }\end{array}$ & Não se aplica \\
\hline \multicolumn{7}{|l|}{$\begin{array}{l}\text { 1. Desastre natural (por exemplo, enchente, furacão, deslizamento de } \\
\text { terra, tornado, terremoto) }\end{array}$} \\
\hline \multicolumn{7}{|l|}{ 2.Incêndio ou explosão } \\
\hline \multicolumn{7}{|l|}{$\begin{array}{l}\text { 3. Acidente com meios de transporte (por exemplo, acidente de carro, } \\
\text { acidente de barco, acidente de trem, acidente de avião) }\end{array}$} \\
\hline \multicolumn{7}{|l|}{ 4. Acidente grave no trabalho, em casa ou durante atividade de lazer } \\
\hline \multicolumn{7}{|l|}{$\begin{array}{l}\text { 5. Exposiç̧ão a substâncias tóxicas (por exemplo, produtos químicos } \\
\text { perigosos, radiação) }\end{array}$} \\
\hline \multicolumn{7}{|l|}{$\begin{array}{l}\text { 6. Agressão física (por exemplo, ser atacado, golpeado, esbofeteado, } \\
\text { chutado, espancado) }\end{array}$} \\
\hline \multicolumn{7}{|l|}{$\begin{array}{l}\text { 7. Agressão com uma arma (por exemplo, ser baleado, esfaqueado, } \\
\text { ameaçado com uma faca, arma, bomba) }\end{array}$} \\
\hline \multicolumn{7}{|l|}{$\begin{array}{l}\text { 8. Agressão sexual (estupro, tentativa de estupro, tentativa de } \\
\text { qualquer tipo de ato sexual através da força ou ameaça) }\end{array}$} \\
\hline \multicolumn{7}{|l|}{ 9. Outra experiência sexual constrangedora ou não consentida } \\
\hline \multicolumn{7}{|l|}{$\begin{array}{l}\text { 10. Combate ou exposição a zonas de guerra (nas forças militares ou } \\
\text { como civil) }\end{array}$} \\
\hline \multicolumn{7}{|l|}{$\begin{array}{l}\text { 11. Cativeiro (por exemplo, ser sequestrado, raptado, ser feito refém, } \\
\text { prisioneiro de guerra) }\end{array}$} \\
\hline \multicolumn{7}{|l|}{ 12. Doença ou lesão com risco de vida } \\
\hline \multicolumn{7}{|l|}{ 13. Sofrimento humano grave } \\
\hline \multicolumn{7}{|l|}{ 14. Morte violenta repentina (por exemplo, homicídio, suicídio) } \\
\hline \multicolumn{7}{|l|}{ 15. Morte acidental repentina } \\
\hline \multicolumn{7}{|l|}{$\begin{array}{l}\text { 16. Ferimentos graves, dano ou morte que você causou a outra } \\
\text { pessoa }\end{array}$} \\
\hline 17. Qualquer outro evento ou experiência gravemente estressantes & & & & & & \\
\hline
\end{tabular}




\section{Parte 2}

A. Se você assinalou o item 17 na PARTE 1, identifique brevemente o evento que você estava pensando:

B. Se você vivenciou mais de um dos eventos da PARTE 1 (itens 1 a 17), pense sobre aquele evento que você considera pior, o qual para este questionário significa 0 evento que atualmente mais incomoda você. Se você vivenciou apenas um dos eventos da PARTE 1, use esse como o pior evento. Por favor, responda às questões seguintes sobre o pior evento (marque todas as opções que se aplicam):

Descreva brevemente o pior evento (por exemplo, o que aconteceu, quem estava envolvido, etc.)

Há quanto tempo isso aconteceu? (por favor, faça uma estimativa se você não tem certeza)

Como você vivenciou este evento?

Aconteceu comigo diretamente

__ Eu testemunhei este evento

__ Eu fiquei sabendo que o evento aconteceu com um membro próximo da família ou amigo próximo

__ Eu fui exposto repetidamente a detalhes deste evento como parte do meu trabalho (por exemplo, paramédico, policial civil, militar ou outro socorrista)

__ Outros, por favor descreva:

A vida de alguém estava em perigo?

__ Sim, minha vida

__ Sim, a vida de outra pessoa

_ Não

\section{Alguém foi gravemente ferido ou morto?}

__ Sim, eu fui gravemente ferido

__ Sim, alguém foi gravemente ferido ou morto

_ Não

Envolveu violência sexual? $\operatorname{Sim}$ Não

Se o evento envolveu a morte de um membro próximo da família ou amigo próximo, foi devido a algum tipo de acidente ou violência, ou foi devido a causas naturais?

_ Acidente ou violência

_ Causas naturais

__ Não se aplica (0 evento não envolveu a morte de um membro próximo da família ou amigo próximo)

Quantas vezes, ao todo, você já experimentou um evento semelhante tão estressante ou quase tão estressante quanto o pior evento?

__ Apenas uma vez

__ Mais de uma vez (por favor, especifique ou estime o número total de vezes que você teve essa experiência) 
Parte 3

Abaixo há uma lista de problemas que as pessoas às vezes apresentam em resposta a uma experiência muito estressante. Pensando em seu pior evento, por favor, leia cuidadosamente cada problema e então circule um dos números à direita para indicar o quanto você tem sido incomodado por este problema no último mês.

\begin{tabular}{|c|c|c|c|c|c|}
\hline No último mês, quanto você foi incomodado por: & $\begin{array}{l}\text { De modo } \\
\text { nenhum }\end{array}$ & Um pouco & $\begin{array}{l}\text { Modera- } \\
\text { damente }\end{array}$ & Muito & Extremamente \\
\hline 1. Lembranças indesejáveis, perturbadoras e repetitivas da experiência estressante? & 0 & 1 & 2 & 3 & 4 \\
\hline 2. Sonhos perturbadores e repetitivos com a experiência estressante? & 0 & 1 & 2 & 3 & 4 \\
\hline $\begin{array}{l}\text { 3. De repente, sentindo ou agindo como se a experiência estressante estivesse, de } \\
\text { fato, acontecendo de novo (como se você estivesse revivendo-a, de verdade, lá no } \\
\text { passado)? }\end{array}$ & 0 & 1 & 2 & 3 & 4 \\
\hline 4. Sentir-se muito chateado quando algo lembra você da experiência estressante? & 0 & 1 & 2 & 3 & 4 \\
\hline $\begin{array}{l}\text { 5. Ter reações físicas intensas quando algo lembra você da experiência estressante } \\
\text { (por exemplo, coração apertado, dificuldades para respirar, suor excessivo)? }\end{array}$ & 0 & 1 & 2 & 3 & 4 \\
\hline $\begin{array}{l}\text { 6. Evitar lembranças, pensamentos, ou sentimentos relacionados à experiência } \\
\text { estressante? }\end{array}$ & 0 & 1 & 2 & 3 & 4 \\
\hline $\begin{array}{l}\text { 7. Evitar lembranças externas da experiência estressante (por exemplo, pessoas, } \\
\text { lugares, conversas, atividades, objetos ou situações)? }\end{array}$ & 0 & 1 & 2 & 3 & 4 \\
\hline 8. Não conseguir se lembrar de partes importantes da experiência estressante? & 0 & 1 & 2 & 3 & 4 \\
\hline $\begin{array}{l}\text { 9. Ter crenças negativas intensas sobre você, outras pessoas ou o mundo (por } \\
\text { exemplo, ter pensamentos tais como: "Eu sou ruim", "existe algo seriamente errado } \\
\text { comigo", "ninguém é confiável", "o mundo todo é perigoso")? }\end{array}$ & 0 & 1 & 2 & 3 & 4 \\
\hline $\begin{array}{l}\text { 10. Culpar a si mesmo ou aos outros pela experiência estressante ou pelo que } \\
\text { aconteceu depois dela? }\end{array}$ & 0 & 1 & 2 & 3 & 4 \\
\hline 11. Ter sentimentos negativos intensos como medo, pavor, raiva, culpa ou vergonha? & 0 & 1 & 2 & 3 & 4 \\
\hline 12. Perder 0 interesse em atividades que você costumava apreciar? & 0 & 1 & 2 & 3 & 4 \\
\hline 13. Sentir-se distante ou isolado das outras pessoas? & 0 & 1 & 2 & 3 & 4 \\
\hline $\begin{array}{l}\text { 14. Dificuldades para vivenciar sentimentos positivos (por exemplo, ser incapaz de } \\
\text { sentir felicidade ou sentimentos amorosos por pessoas próximas a você)? }\end{array}$ & 0 & 1 & 2 & 3 & 4 \\
\hline 15. Comportamento irritado, explosões de raiva ou agir agressivamente? & 0 & 1 & 2 & 3 & 4 \\
\hline 16. Correr muitos riscos ou fazer coisas que podem Ihe causar algum mal? & 0 & 1 & 2 & 3 & 4 \\
\hline 17. Ficar "super" alerta, vigilante ou de sobreaviso? & 0 & 1 & 2 & 3 & 4 \\
\hline 18. Sentir-se apreensivo ou assustado facilmente? & 0 & 1 & 2 & 3 & 4 \\
\hline 19. Ter dificuldades para se concentrar? & 0 & 1 & 2 & 3 & 4 \\
\hline 20. Problemas para adormecer ou continuar dormindo? & 0 & 1 & 2 & 3 & 4 \\
\hline
\end{tabular}

